\title{
Unusual Root Staining of the Third Molars in a Patient Exposed to Lead and Tetracycline
}

\section{Raid Sadda ${ }^{1 *}$ and Ayad Sadda ${ }^{2}$}

${ }^{1}$ Department of Oral and Maxillofacial Surgery, College of Dentistry, New York University, USA

${ }^{2}$ Ross University School of Medicine, USA

\begin{abstract}
Purpose: The purpose of this case report is to evaluate a root stain, and to determine its composition when an individual has been exposed to multiple factors. The main goal of this particular study is to determine if it is the use of tetracycline or the exposure to lead which may have caused the staining of multiple roots.

Introduction: Undetermined root-staining in a 21 year old male exposed to multiple environmental factors; specifically lead and tetracycline.

Discussion and conclusion: A history of exposure to multiple contaminants, such as lead and tetracycline, may obscure the accuracy of a diagnosis, especially when it is well-known that these particular contaminants stain the root of an individual's teeth in a similar fashion. Patients with stained teeth and a history of exposure to environmental or ingested potentially staining or noxious elements (like lead) should have a blood test to determine its presence, and further be treated for level-specific quantities.
\end{abstract}

Keywords: Lead exposure; Tetracycline; Third-molar root staining

\section{Introduction}

Intrinsic staining of teeth can result from introducing a variety of substances into the body which can form complexes with hard tissues (enamel, dentine, and bone). Two well-known substances that can cause such intrinsic staining of the teeth are lead and tetracyclineclass medications $[1,2]$. Lead has been used for centuries in many industrial processes such as the development of batteries, sheets for $\mathrm{X}$-Ray films, protective gear for high-radiation purposes (lead doors and lead gowns), and more stigmatically in the past, gasoline and paint. Lead poisoning occurs when a person of any age swallows, absorbs, or inhales lead in any form $[3,4]$. Tetracycline and some of its homologues, such as minocycline, have a tendency to deposit in the hard substance of tooth and bone by forming a tetracycline-calcium-orthophosphate complex, which produces an intrinsic stain $[5,6]$. The intensity of the stain depends on the dosage, duration of treatment, and age of onset of treatment. The age of onset of the treatment using tetracycline based medications is also important in determining the pattern of staining, such as the stratification of staining of the $\operatorname{root}(\mathrm{s})[1,7,8]$.

\section{Case Report}

A 21-year-old Caucasian male with a history of acne treated with a nine-month course of tetracycline at the age of 16 , presents to the dental office by referral for the extraction of all third-molar teeth. Informed consent was obtained from the patient. On oral examination, the gingiva, teeth, and surrounding tissue appeared normal (Figure 1). A panoramic X-Ray of the patient's maxilla and mandible appeared normal and unperturbed (Figure 2). Upon extraction, a visible black band was present on the middle-third of each root on each tooth. These bands were uniform in appearance and the surrounding enamel was normal in color (Figure 3). The tooth was sent for histopathology, crosssectioned, and stained with fluorescence. The results were remarkable for tetracycline-staining and stratification (Figure 4). Upon further questioning of the patient's occupational history, he states that he has been working in a lead factory for 3 years, manufacturing lead foil for dental films as well as lead-based doors serving as X-Ray barriers. This prompted a referral to the patient's primary care physician to screen the patient for a blood-lead level. The result of the blood screen reported to be within normal limits (Range: $\mathrm{Pb}$ adult $<20 \mathrm{mcg} / \mathrm{dL}$; child $<5 \mathrm{mcg}$ / $\mathrm{dL})$.

\section{Discussion}

Enamel formation of the third molars takes place between the age of 12 and 16, while root formation takes place between the age of 18 and 25 [9]. A history of exposure to multiple contaminants, such as lead and tetracycline, may obscure the accuracy of a diagnosis, especially when it is well-known that these particular contaminants stain the root of an individual's teeth in a similar fashion. In some cases, lead is visibly deposited at the cervical line of the teeth (Figure 4). This is

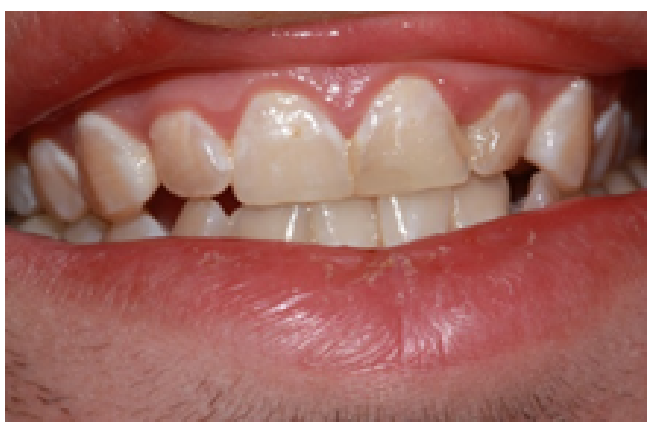

Figure 1: Enamel of the teeth in a 21 year-old male receiving tetracycline and minocycline therapy for Acne vulgaris.

*Corresponding author: Raid Sadda, Department of Oral and Maxillofacia Surgery, College of Dentistry, New York University, USA, Tel: 212-998-9660; E-mail: rs211@nyu.edu

Received January 06, 2015; Accepted February 26, 2015; Published March 03 2015

Citation: Sadda R, Sadda A (2015) Unusual Root Staining of the Third Molars in a Patient Exposed to Lead and Tetracycline. Dentistry 5: 286. doi:10.4172/21611122.1000286

Copyright: ( 2015 Sadda R, et al. This is an open-access article distributed under the terms of the Creative Commons Attribution License, which permits unrestricted use, distribution, and reproduction in any medium, provided the original author and source are credited. 
Citation: Sadda R, Sadda A (2015) Unusual Root Staining of the Third Molars in a Patient Exposed to Lead and Tetracycline. Dentistry 5: 286. doi:10.4172/2161-1122.1000286

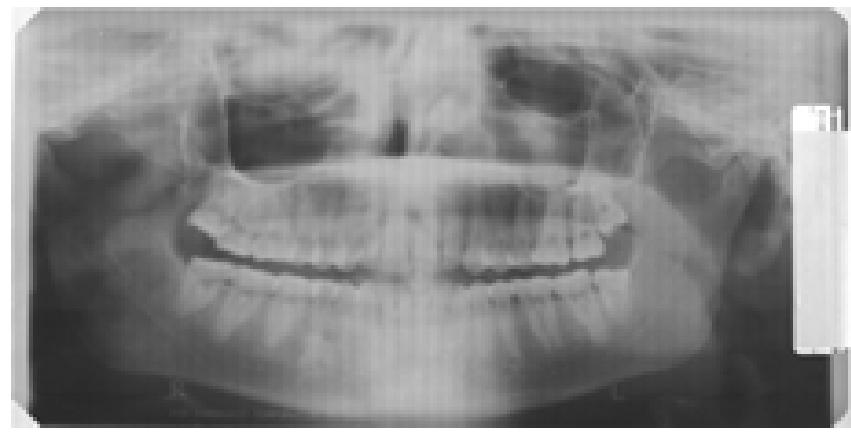

Figure 2: Panoramic X-ray of same patient in figure 3 revealed uncompromised bone structures.

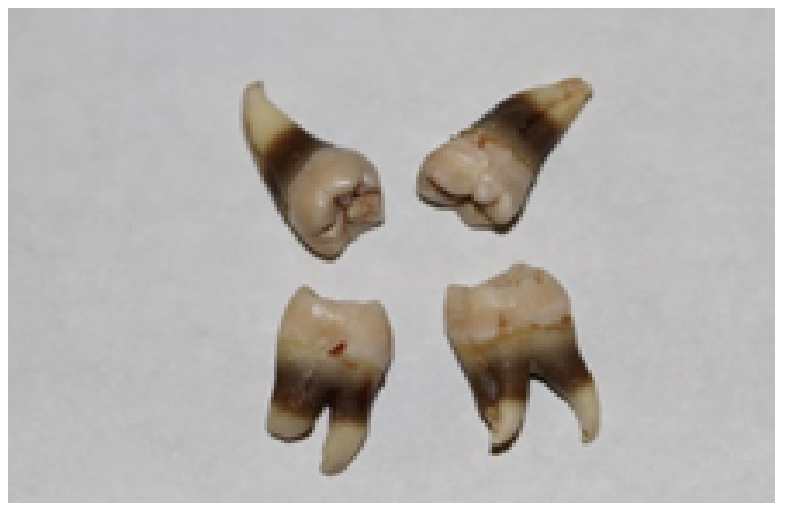

Figure 3: Four recently extracted third molar teeth from 21- year old man who had been taken tetracycline and minocycline at the age of 16 . The discoloration is at the middle third of the root.

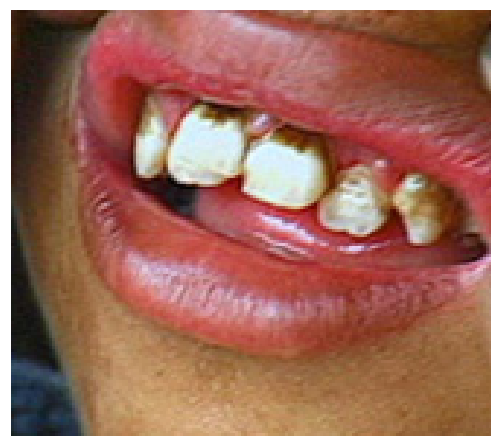

Figure 4: Black staining of the teeth due to lead exposure (from village of the black teeth, S. Allen Counter).

caused by long-term exposure to lead or lead-based products $[2,10,11]$. Tetracycline and its analogs, including minocycline, are known to cause a similar pattern of stratification in the roots of the teeth, which further depends on the age of onset during which the administration of these medications took place $[5,6,8]$ (Figure 5 and 6 ). The presence of banding on extracted teeth warrants further investigation via history taking followed by screening for lead toxicity. With the presence of occupational exposure to lead, and a long history of acne treated with tetracycline and minocycline combinations, differentiation of the composure can be made difficult. However, through histological sampling to visualize stratifications (seen with tetracycline exposure,
Figure 4) and in vitro reagent sampling for lead in the extracted teeth of adults [2,11]; (Figure 4); differentiating between the compositions of the staining pattern is made possible. Tetracycline is known to chelate calcium and thus is incorporated in the hydroxyapatite crystals within the teeth in a lamellar fashion, which can then be tagged with ultraviolet-sensitive substrate to be seen under a microscope [5]; (Figure 4). The patient in the case presented was treated at the age of 16 , which falls into the categorical age by which the root develops, and therefore incorporated the tetracycline in the mentioned region of the tooth. Nevertheless, it is not necessary to have structural abnormalities, as is evidenced by the patient's panoramic X-Ray (Figure 2). Having the tools to help distinguish which contaminant is responsible for the particular root staining may open a new tenet for a narrower management plan that is specific to those particular environmental contributors.

\section{Conclusion}

The structural integrity of the teeth seen in the patient was unaffected, regardless of his past use of tetracycline and minocycline, as well as his 3-year history of an occupational exposure to lead. While there is dental evidence which suggests an exposure to an unknown substance, it is important to have quality history taking skills to understand which exposure, whether intentional (medication) or unintentional (occupation) that the patient is aware of, and how this affects him or her. Advanced measures may include a screening test to rule out

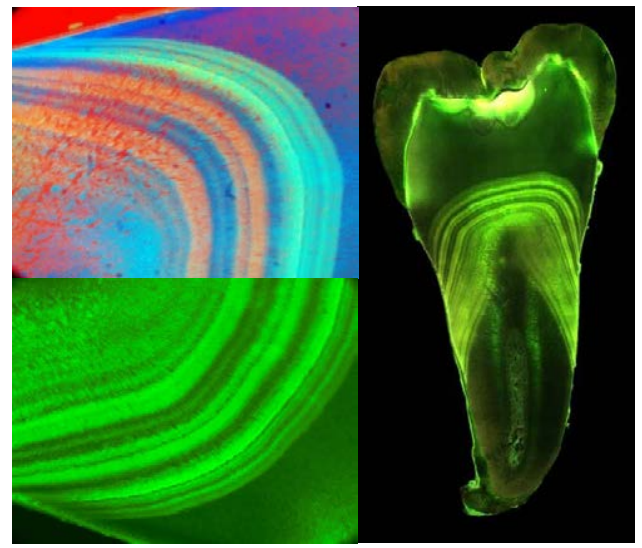

Figure 5: Cross-section of tetracycline-stained wisdom tooth taken with ultraviolet light (courtesy of Dr. Timothy Bromage, Ph.D.).

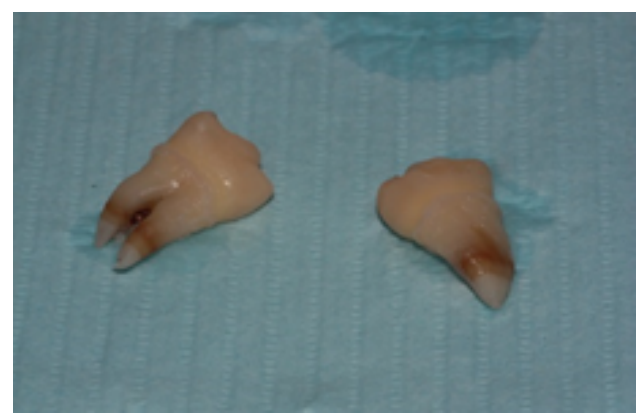

Figure 6: Two recently extracted Maxillary and Mandibular third molar teeth from a 22 year-old female who had been taken tetracycline and minocycline for Acne vulgaris at age 18. The discoloration of the root is at the apical third of the root. 
Citation: Sadda R, Sadda A (2015) Unusual Root Staining of the Third Molars in a Patient Exposed to Lead and Tetracycline. Dentistry 5: 286. doi:10.4172/2161-1122.1000286

asymptomatic blood-lead toxicity, as well as other methods after tooth extraction to determine past exposures which where subclinical at the time of examination. This includes lead-reagent testing of root and enamel, and histo-analysis of photosensitive tetracycline biomarkers. Patients with stained teeth and a history of exposure to environmental or ingested potentially staining or noxious elements (like lead) should have a blood test to determine its presence.

\section{References}

1. Wallman IS, Hilton HB (1962) Teeth pigmented by tetracycline. Lancet 1: 827829.

2. Shepherd TJ, Dirks W, Manmee C, Hodgson S, Banks DA, et al. (2012) Reconstructing the life-time lead exposure in children using dentine in deciduous teeth. Sci Total Environ 425: 214-222.

3. Rebich T Jr, Kumar J, Brustman B (1983) The st. Regis environmental health issue. Assessment of dental defects. JADA 106: 630-633.

4. http://www.cdc.gov/nceh/lead/tips/sources.htm
5. Davies AK, Cundall RB, Dandiker Y, Slifkin MA (1985) photo-oxidation of tetracycline adsorbed on hydroxyapatite in relation to light-induced staining of teeth. J Dent-Res 64: 936-939.

6. Fenske NA, Millns JL (1980) Cutaneous pigmentation due to minocycline hydrochloride. J Am Acad Dermatol 3: 308-310.

7. Cohen BD, Abrams BL (1889) Unusual case of stained roots of unerupted third molars. Gen Dent 37: 342-343.

8. McKenna BE, Lamey PJ, Kennedy JG, Bateson J (1999) Minocycline-induced staining of the adult permanent dentition: A review of the literature and Report of a case. Dent Update 26: 160-162.

9. Wheeler RC (1974) Dental Anatomy, physiology and occlusion. (5thedn), Philadelphia, Saunders, pp: 30.

10. Allen Counter S (2007) Village of the black teeth. The Boston Globe newspaper.

11. Paul M (1992) Chapter 3 The Monitoring of Lead Exposure. Human Lead Exposure BY Herbert L. Needleman. Boca Raton: CRC. 